\title{
Polypharmacy and excessive polypharmacy in community-dwelling middle aged and aged adults between 2011 and 2015
}

\author{
Laura Hellemans $^{1}$ (D) | Shauni Nuyts ${ }^{2,3}$ | Julie Hias ${ }^{1}$ | Marjan van den Akker,4,5 | \\ Gijs Van Pottelbergh $^{2}$ | Xavier Rygaert ${ }^{6}$ | Isabel Spriet ${ }^{1,7}$ | Bert Vaes ${ }^{2}$ | Jos Tournoy ${ }^{8,9}$ | \\ Lorenz Van der Linden ${ }^{1,7}$
}

\author{
${ }^{1}$ Pharmacy Department, University \\ Hospitals Leuven, Leuven, Belgium \\ ${ }^{2}$ Academic Centre of General Practice/ \\ Department of Public Health and Primary \\ Care, KU Leuven, Leuven, Belgium \\ ${ }^{3}$ L-BioStat, KU Leuven, Leuven, Belgium \\ ${ }^{4}$ Institute of General Practice, Johann \\ Wolfgang Goethe University, Frankfurt, \\ Germany \\ ${ }^{5}$ Department of Family Medicine, School \\ CAPHRI, Maastricht University, Maastricht, \\ The Netherlands \\ ${ }^{6}$ InterMutualistic Agency (IMA), Brussels, \\ Belgium \\ ${ }^{7}$ Department of Pharmaceutical and \\ Pharmacological Sciences, KU Leuven, \\ Leuven, Belgium \\ ${ }^{8}$ Department of Geriatric Medicine, \\ University Hospitals Leuven, Leuven, \\ Belgium \\ ${ }^{9}$ Department of Chronic Diseases, \\ Metabolism and Ageing, KU Leuven, Leuven, \\ Belgium
}

\section{Correspondence}

Laura Hellemans, Pharmacy Department, University Hospitals Leuven, Herestraat 49, 3000 Leuven, Belgium.

Email: laura.hellemans@uzleuven.be

\begin{abstract}
Aims: We aimed to assess the prevalence, components and evolution of polypharmacy and to evaluate risk factors associated with polypharmacy.

Methods: A retrospective dynamic cohort study was performed, using a primary healthcare database comprising Flemish community-dwelling adults aged $\geq 40$ years between 2011 and 2015. Polypharmacy and excessive polypharmacy were defined as the use of 5-9 or minimum 10 different medications during 1 year, respectively. Temporal changes were analysed using an autoregressive error model. Risk factors for polypharmacy were evaluated using logistic regression.

Results: In total, 68426 patients were included in the analysis. The prevalence of polypharmacy was $29.5 \%$ and $16.1 \%$ for excessive polypharmacy in 2015 . The agestandardised prevalence rate of patients using minimum five medications increased with $1.3 \%$ per year (95\% confidence interval (Cl): 0.1968-2.4279). The mean number of unplanned hospital admissions was 0.07 (standard deviation (SD) 0.33 ) for polypharmacy patients and 0.19 (SD 0.53) for excessive polypharmacy patients. Four risk factors were found to be significantly correlated with polypharmacy: age (odds ratio (OR) 1.015; 95\% Cl: 1.013-1.017), female gender (OR 1.161; 95\% Cl: 1.108-1.216), number of chronic diseases (OR 1.126; $95 \% \mathrm{Cl}$ : 1.114-1.139) and number of general practitioner contacts (OR 1.283; 95\% Cl: 1.274-1.292).

Conclusion: The prevalence of polypharmacy increased between 2011 and 2015. Polypharmacy and excessive polypharmacy patients appeared to differ based on our observations of characteristics, drug therapy and outcomes. Age, female gender, number of chronic diseases and number of general practitioner contacts were associated with polypharmacy.
\end{abstract}

\section{1 | INTRODUCTION}

Polypharmacy has been considered a harbinger of potentially avoidable harm in adults. ${ }^{1}$ The concomitant intake of at least 5 or 10 medications are currently the most commonly applied

Laura Hellemans and Shauni Nuyts are the first authors as they contributed equally to this paper. definitions for polypharmacy and excessive polypharmacy, respectively. ${ }^{1,2}$ Given the high prevalence of multimorbidity, polypharmacy has become nearly inevitable in a large proportion of adults. ${ }^{3}$ Risk factors for polypharmacy are diverse and have already been reported on extensively. Both patient-related factors such as older age, female gender and multimorbidity and healthcare-related factors such as multiple prescribers are all known to 
be associated with polypharmacy. ${ }^{4-6}$ Yet, a consensus on a working definition of polypharmacy has not been reached. Most commonly, an arbitrary definition of concomitant use of five or more medications is followed. ${ }^{2}$

The clinical impact of polypharmacy by virtue of its association with unplanned and/or drug-related hospital admissions cannot be understated. ${ }^{7}$ This association seems to be age dependent; in older adults, medication-related harm (MRH) has even been identified as a common geriatric syndrome. ${ }^{1,8,9}$ Multiple strategies have subsequently been investigated to improve clinical outcome by specifically targeting polypharmacy. A majority of trials that were performed in primary healthcare have, however, failed to show any clinically significant improvement or to impact patient-related outcomes. ${ }^{10}$ Furthermore, most positive clinical trials have been performed in the hospital setting, and as a result in a minority and highly selected sample of all older adults. ${ }^{11}$

Disappointing results of trials in primary healthcare might be in part due to the enrolment of low-risk or clinically stable patients or even owing to a lack of a causal relation between polypharmacy and clinical outcome. The latter might be in its turn attributed to the use of suboptimal polypharmacy definitions. Appropriateness of polypharmacy cannot always be evaluated, and many patients will meet the minimum intake of five medications, but not all will be at increased risk for medication harm. ${ }^{8,12}$ The term excessive polypharmacy has been introduced in an attempt to further distinguish patients with very high medication use. ${ }^{2}$ However, potential differences between polypharmacy and excessive polypharmacy patients have not yet been fully investigated. More in-depth knowledge on patient characteristics and medication use, risk factors and temporal changes across age categories is hence warranted to better comprehend the polypharmacy population in primary healthcare.

Therefore, we aimed to determine the prevalence and evolution of polypharmacy in middle aged and aged adults in a large dynamic primary healthcare cohort. Furthermore, we described medication use, patient characteristics and outcomes in both the total dynamic cohort and polypharmacy subgroups. Finally, risk factors for polypharmacy were evaluated.

\section{2 | METHODS}

\section{1 | Study setting and design}

A retrospective dynamic cohort study was conducted. Data from the linked Intego and InterMutualistic Agency (IMA) database were collected. ${ }^{13,14}$ Intego is a Flemish general practice-based morbidity registration network governed by the Academic Centre of General Practice of the KU Leuven-University of Leuven. The collected data concerned a dynamic representative sample of the Flemish population, which varies annually between $1.5 \%$ and $2 \%$ of all Flemish civilians and is based on their National Insurance Number. Intego provided information on electronic prescriptions, diagnoses, laboratory results and clinical parameters. ${ }^{13}$ IMA collected administrative

\section{What's known}

- Polypharmacy is associated with medication-related harm and other negative clinical outcomes.

- Although the consequences of polypharmacy have been extensively described, interventions to treat polypharmacy still fail to impact patient-related and clinical outcomes.

\section{What's new}

- The prevalence of polypharmacy and excessive polypharmacy increased significantly in patients aged $\geq 75$ years.

- Polypharmacy and excessive polypharmacy patients seemed to differ based on patient characteristics, drug therapy and outcomes. This emphasises the importance of further investigating differences in both patient populations.

- Our results on patient characteristics and drug therapy will contribute to future research on optimising underachieving polypharmacy interventions by better defining target populations.

data such as year of death and health expenditure data (eg, hospital admissions, emergency department visits, dialysis) of all Belgian inhabitants. For this analysis, IMA provided data for Intego patients only. Furthermore, this study is part of the larger PriMed research project on identifying patients with the highest risk for unplanned hospital admissions in middle aged and aged community-dwelling adults.

\section{2 | Patient population}

Intego data were available from 1994 to 2015. IMA data were available from 2011 to 2015 and for patients aged at least 40 years. As Intego is a dynamic cohort, patients were divided into annual contact groups with the majority of patients being included at the start of a year and a minority of the patients during the year. For example, patients with a first contact in Intego before 2011, a last contact in or after 2011 and aged at least 40 years on January 1, 2011 were included in the annual contact group of 2011 at the start of the year. Patients who had their first contact in 2011 and were at least 40 years old in 2011 were included during the year. For the next year, patients who died (defined as 'deaths') or patients who had their last contact with Intego (defined as 'lost contact') in 2011 were excluded. This was repeated for each consecutive year up to 2015. Patients who stayed in a nursing home between 2011 and 2015 were excluded from the study as Electronic Health Records (EHR) in nursing homes were not registered systemically in Intego. 


\section{3 | Polypharmacy subgroups}

Patients were categorised into three groups according to the total number of different prescribed medications for that specific year and counted at level 4 of the Anatomic Therapeutic Chemical (ATC) classification. ATC4 was chosen to prevent medication switches within a class (eg, pantoprazole to omeprazole) from being counted as different medications. Concomitant usage was not taken into account. Non-polypharmacy (nPP) patients received prescriptions for maximum four different medications per year. Polypharmacy $\left(\mathrm{PP}_{5-9}\right)$ patients were prescribed minimum five and maximum nine different medications per year. In excessive polypharmacy $\left(\mathrm{PP}_{10+}\right)$ patients, at least 10 different medications per year had to be registered. The terms $\mathrm{nPP}, \mathrm{PP}_{5-9}$ and $\mathrm{PP}_{10+}$ were used when referring to patient groups.

\section{4 | Data collection}

The following data were collected from the linked database to describe medication use and patient characteristics in the total dynamic cohort for the years 2011-2015: age; sex; number of general practitioner (GP) contacts in Intego, counted as the total number a patient's EHR was opened by the GP; overall mortality rate; number of all-cause hospital admissions; number of unplanned hospital admissions, defined as an emergency department (ED) visit immediately followed by hospitalisation; number of all-cause ED visits; number of chronic diseases and the prevalence of the 10 most common chronic diseases.

The list of chronic diseases was based on an adapted version of the list of Knottnerus JA et al, who used the International Classification of Primary Care-1st edition (ICPC-1) codes and is available as online Supporting Information. ${ }^{15}$ The following chronic diseases were analysed as a group. All cancer-related diseases were analysed as the group "Malignancies," femur fracture (L75) and osteoporosis (L95) were only counted as a chronic disease if both were present, 10 psychological chronic diseases were analysed as the group "Psychological conditions," three types of anaemia were analysed as the group "Anaemia." The order of the 10 most common chronic diseases was based on data collected from the annual contact group of 2011. If the top 10 of another year included different chronic diseases than 2011, the prevalence of these chronic diseases was reported for each year.

Medication use was measured by counting the prescriptions for different medications as registered in the Intego database and was based at ATC4 level. Additionally, the prevalence of the 10 most prescribed medications was collected. The order was based on the top 10 of 2011. If the top 10 of another year included different medications than 2011, the prevalence of these medications was reported for each year. For the analysis of the most used medications, the following ATC codes were analysed as the group 'Non-Steroidal AntiInflammatory Drugs' (NSAIDs): M01AB, M01AC, M01AE, M01AH and M01AX.
For the year 2015, the same data on medication use and patient characteristics as for the total dynamic cohort were used for each polypharmacy group (ie, $\mathrm{nPP}, \mathrm{PP}_{5-9}$ and $\mathrm{PP}_{10+}$ ), except for the number of medications. The annual contact group of 2015 comprised the most recent data and was therefore chosen for the medication analysis. We reported the order of the 10 most common chronic diseases and 10 most prescribed medications based on the $\mathrm{PP}_{10+}$ group. If the top 10 of another group included different chronic diseases or medications than the $\mathrm{PP}_{10+}$ group, the prevalence of these chronic diseases and medications were then shown for each group.

Data on all hospital contacts were drawn from the IMA dataset; the remainder of the data was provided by the Intego database.

\section{5 | Statistical analysis}

Standard descriptive statistics were used for patient characteristics. Normality was visually assessed. Normally distributed variables were characterised by mean ( \pm standard deviation (SD)). Skewed data were reported as median (interquartile range (IQR) Q1-Q3). Proportions and counts were represented as n (\%). Age was reported as a continuous variable and divided into three age categories: $40-64$ years, $65-74$ years and a minimum age of 75 years.

We performed two analyses to evaluate temporal changes of polypharmacy. We used an autoregressive error model as we assumed data were correlated (PROC AUTOREG in SAS). To specify, a stepwise autoregression was applied where a higher-order model was fitted with multiple autoregressive lags. In this procedure, autoregressive parameters were sequentially removed until the remaining autoregressive parameters had significant $t$ tests. ${ }^{16}$ In the first analysis, $\mathrm{PP}_{5-9}$ and $\mathrm{PP}_{10+}$ patients were analysed together as $\mathrm{PP}_{5+}$ patients. $^{2}$ The prevalence rates for $\mathrm{nPP}$ and $\mathrm{PP}_{5+}$ were age standardised with the Flemish population of the year 2000 as reference group and reported with $P$ value (statistical significance at $<.05)$ and $95 \%$ confidence interval $(\mathrm{Cl})$. In the second analysis, more details were provided for each of the subgroups and the prevalence rates were thus calculated for $\mathrm{nPP}, \mathrm{PP}_{5-9}$ and $\mathrm{PP}_{10+}$ patients per age category.

We also evaluated the association between polypharmacy and age, sex, number of chronic diseases and number of GP contacts. The selection of variables was based on previous research and availability in our database. ${ }^{4,5,17}$ Only risk factors with a $P$ value $<.05$ in the univariate analysis were included in the multivariable analysis. Multivariable logistic regression was used to analyse the association between the selected risk factors and polypharmacy. ${ }^{18}$ Here, $\mathrm{PP}_{5-9}$ and $\mathrm{PP}_{10+}$ patients were again analysed together as $\mathrm{PP}_{5+}$ patients. ${ }^{2}$ Variables were derived from 2014 and polypharmacy status was assessed in 2015. Hence, only patients with data available for both years were included in this analysis. The year 2015 comprised the most recent data and was therefore chosen for this analysis. A model fit was assessed using the likelihood ratio test, score test and Wald 
test. Statistical analysis was performed using SAS software (PROC LOGISTIC).

\subsection{Ethics approval}

The integration of both databases was approved by the Sectoral Committee of Social Security and Health (no. 17/071). This study was part of a research project which was approved by the Ethics Committee of KU Leuven (S61973). All data were pseudonymised and the authors did not have access to identifiable patient information. The study conforms to the standards of the Declaration of Helsinki.

\section{3 | RESULTS}

Data from 68426 patients were included in the analysis. The number of patients in each annual contact group is shown in Figure 1. Data from five consecutive years were collected: $2011(n=41334)$, 2012 ( $n=43$ 396), 2013 ( $n=45$ 038), 2014 ( $n=46$ 131) and 2015 ( $n=43285)$.

\section{1 | Total dynamic cohort}

Characteristics of the total dynamic cohort are summarised in Table 1. Normality assumptions were visually ascertained and met. The median age was 56 years (IQR 48-66) in 2011 and 58 years (IQR 49-69) in 2015. The proportion of patients aged 75 years and older was $13 \%$ in 2011 and $16 \%$ in 2015 . The proportion of men remained unaltered across the annual contact groups (49\% in 2011-2014 and 48\% in 2015) as did the overall mortality rate (10.19/1000 in 2011 and 10.26/1000 in 2015). The median number of GP contacts was 3 (IQR 1-7) in 2011 and 4 (IQR 2-8) in 2015. For all-cause hospital admissions, a mean of 0.43 (SD 1.37) in 2011 and 0.50 (SD 1.49) in 2015 was observed. Uncomplicated hypertension remained the most prevalent chronic disease in each year (21.2\% in 2011 and $23.9 \%$ in 2015). The median number of prescribed medications was 3 (IQR 0-7) in 2011 and 4 (IQR 1-8) in 2015. In all annual contact groups, NSAIDs were prescribed most commonly with a percentage of $26.6 \%$ in 2011 and $30.2 \%$ in 2015 .

\subsection{Evolution of polypharmacy}

Temporal trends of polypharmacy between 2011 and 2015 across all age categories have been depicted in Figure 2. Following the stepwise autoregression, none of the autoregressive components were statistically significant indicating a very weak autocorrelation between years.

The age-standardised prevalence rate of $\mathrm{PP}_{5+}$ increased significantly with $1.3 \%$ per year ( $95 \% \mathrm{Cl}$ : 0.1968 to $2.4279, P=.0333$ ) (Figure 2A). Figure 2B depicts the proportion and prevalence rates

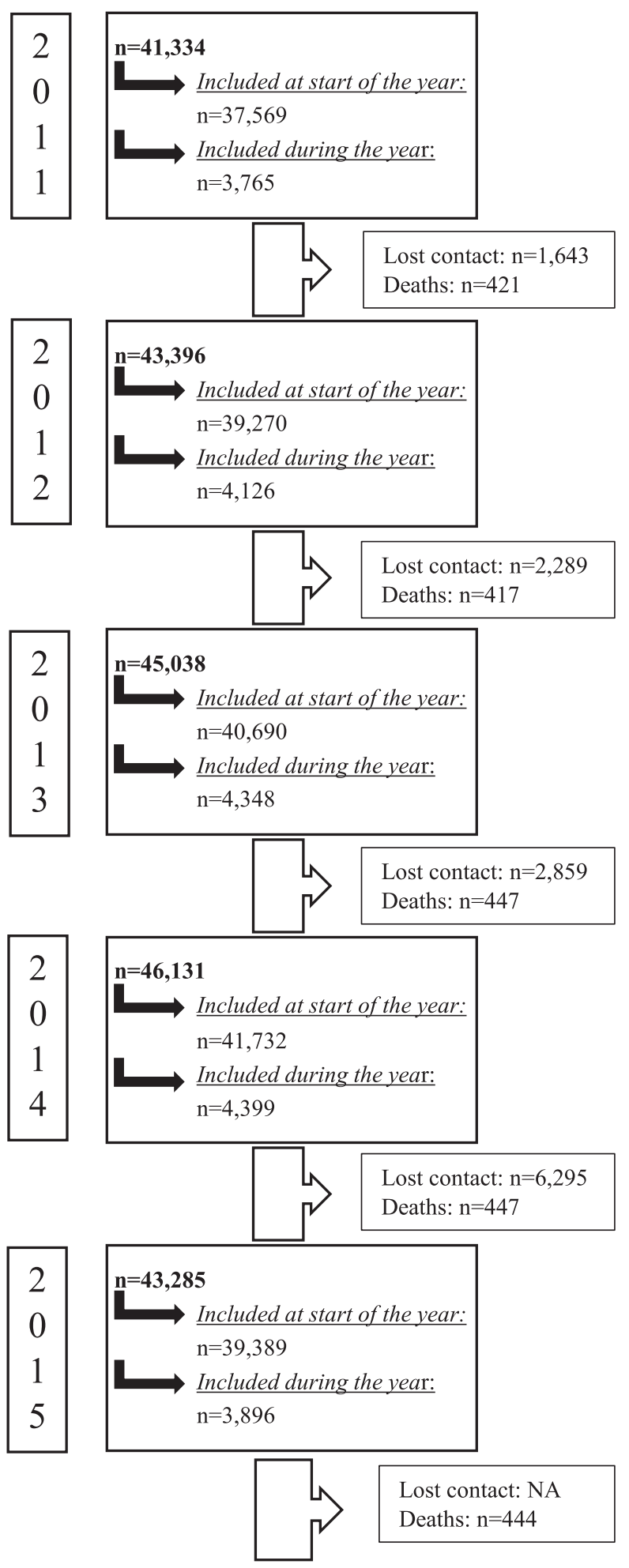

FIGURE 1 Flow chart of annual contact groups from 2011 to 2015. NA, not applicable

of $\mathrm{nPP}, \mathrm{PP}_{5-9}$ and $\mathrm{PP}_{10+}$ patients per age category for each year. The age category of 65-74 years comprised $32 \% \mathrm{PP}_{5-9}$ patients in 2011 and $36 \% \mathrm{PP}_{5-9}$ patients in 2015. In 2011, 21\% of the patients aged 65-74 years were considered $\mathrm{PP}_{10+}$ patients and this was $24 \%$ in 2015. In the age category 75 years and older, $24 \%$ of patients were considered $\mathrm{PP}_{10+}$ patients in 2011, this was $28 \%$ in 2015. In the nPP group, the 
TABLE 1 Description of the total dynamic cohort per annual contact group

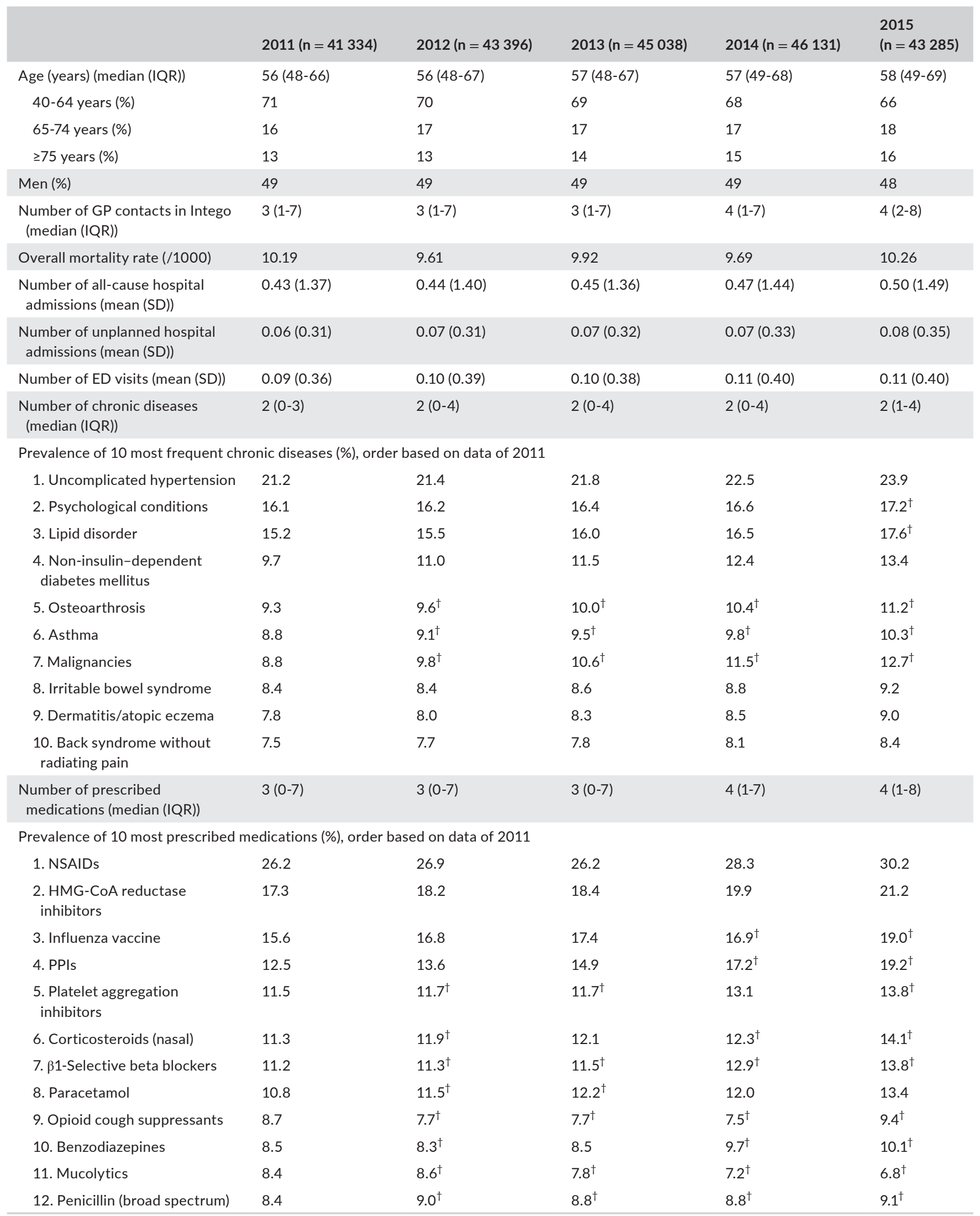

Note: Values indicated with ${ }^{\dagger}$ are not on their correct position in top 10 according to their prevalence in that respective year.

Abbreviations: ED, Emergency Department; GP, general practitioner; HMG-CoA reductase, 3-hydroxy-3-methyl-glutaryl-CoA reductase; IQR, interquartile range; NSAIDs, non-steroidal anti-inflammatory drugs; PPIs, proton pump inhibitors; SD, standard deviation. 
A Age-standardized prevalence rates for polypharmacy

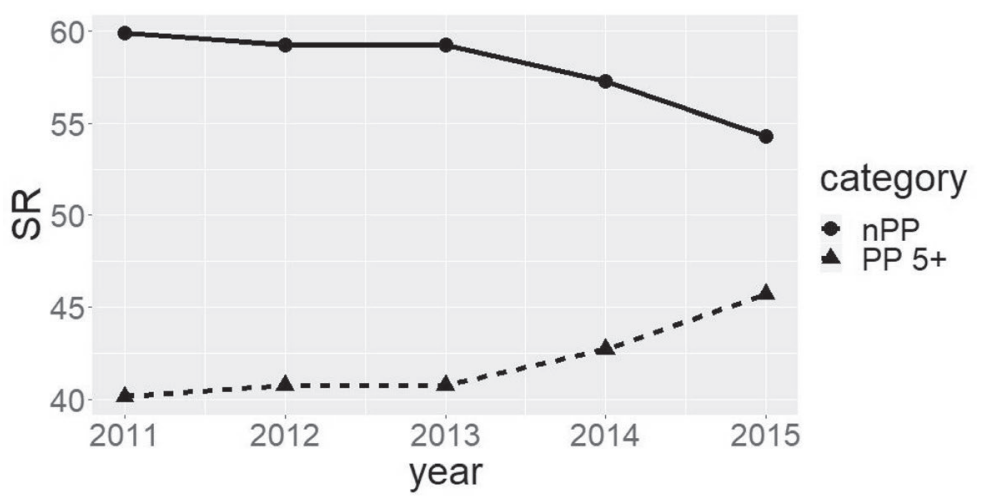

\begin{tabular}{|c|c|c|c|}
\hline Category & Estimate (year) & $\boldsymbol{P}$-value & $\mathbf{9 5 \%}$ CI \\
\hline $\mathbf{n P P}$ & -1.3124 & $0.0333^{*}$ & {$[-2.4279 ;-0.1968]$} \\
\hline $\mathbf{P P}_{5+}$ & 1.3124 & $0.0333^{*}$ & {$[0.1968 ; 2.4279]$} \\
\hline
\end{tabular}

B Proportion of $n P P, P P_{5-9}$ and $P P_{10+}$ patients per age category for each year

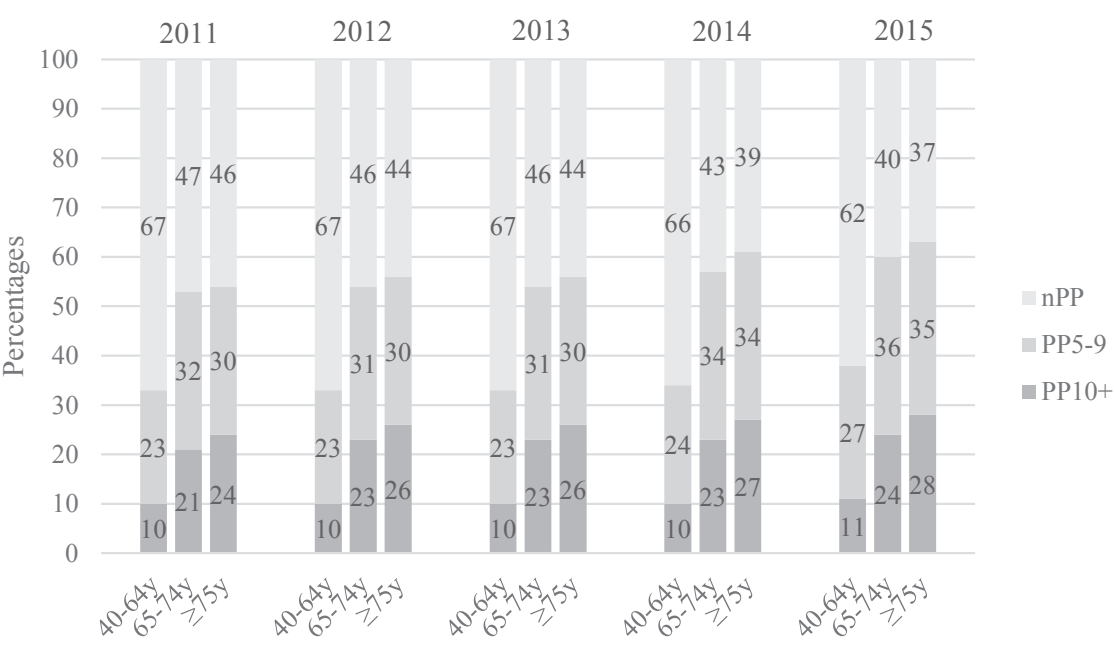

\begin{tabular}{|c|c|c|c|c|c|}
\hline Category & Age (years) & Estimate (year) & $\boldsymbol{P}$-value & 95\% CI lower & 95\% CI upper \\
\hline $\mathrm{nPP}$ & $40-64$ & -0.99300 & 0.0845 & -2.23600 & 0.25000 \\
\hline $\mathrm{nPP}$ & $65-74$ & -1.63800 & $0.0231^{*}$ & -2.84953 & -0.42647 \\
\hline $\mathrm{nPP}$ & $75+$ & -2.31700 & $0.0137^{*}$ & -3.72995 & -0.90405 \\
\hline $\mathrm{PP}_{5-9}$ & $40-64$ & 0.79200 & 0.0853 & -0.20350 & 1.78750 \\
\hline $\mathrm{PP}_{5-9}$ & $65-74$ & 1.10700 & 0.0802 & -0.24682 & 2.46082 \\
\hline $\mathrm{PP}_{5-9}$ & $75+$ & 1.44000 & $0.0192 *$ & 0.44623 & 2.43000 \\
\hline $\mathrm{PP}_{10+}$ & $40-64$ & 0.20000 & 0.2555 & -0.25405 & 0.65405 \\
\hline $\mathrm{PP}_{10+}$ & $65-74$ & 0.53000 & 0.0747 & -0.09807 & 1.15807 \\
\hline $\mathrm{PP}_{10+}$ & $75+$ & 0.88100 & $0.0167 *$ & 0.30268 & 1.45932 \\
\hline
\end{tabular}

FIGURE 2 Temporal changes of polypharmacy. $\mathrm{Cl}$, confidence interval; nPP, non-polypharmacy; $\mathrm{PP}_{5+}$, polypharmacy defined as the intake of minimum five medications; $\mathrm{PP}_{5-9}$, polypharmacy defined as the intake of minimum five and maximum nine medications; $\mathrm{PP}_{10+}$, excessive polypharmacy; SR: standardised rates; $P$ values indicated with * are statistically significant proportion of patients aged 65-74 years decreased significantly with $1.6 \%$ per year ( $95 \% \mathrm{Cl}:-2.84953$ to $-0.42647, P=.0231$ ). For patients aged 75 years and older, a significant decrease for nPP with $2.3 \%$ per year (95\% Cl: -3.72995 to $-0.90405, P=.0137$ ) was observed. For the $\mathrm{PP}_{5-9}$ and $\mathrm{PP}_{10+}$ groups, the proportion of patients aged 75 years or older increased significantly with $1.4 \%$ (95\% Cl: 0.44623 to 2.43000 , $P=.0192)$ and $0.9 \%$ per year (95\% Cl: 0.30268 to $1.45932, P=.0167$ ), respectively. Other results were not significant (Figure $2 \mathrm{~B}$ ).

\subsection{Polypharmacy and excessive polypharmacy}

Table 2 shows the medication use and patient characteristics categorised according to the PP groups: $\mathrm{nPP}, \mathrm{PP}_{5-9}$ and $\mathrm{PP}_{10+}$ patients. In 2015, the nPP group included 23549 patients (54.4\%), the $\mathrm{PP}_{5-9}$ group 12779 patients (29.5\%) and the $\mathrm{PP}_{10+}$ group 6957 patients (16.1\%). In the nPP group, the vast majority (76\%) of patients was younger than 65 years. The total proportion of all patients aged 
TABLE 2 Description of the polypharmacy groups in 2015

\begin{tabular}{|c|c|c|c|}
\hline & $n P P(n=23549)$ & $\mathrm{PP}_{5-9}(\mathrm{n}=12779)$ & $\begin{array}{l}\mathrm{PP}_{10+} \\
(\mathrm{n}=6957)\end{array}$ \\
\hline Age (years) (median (IQR)) & $54(47-64)$ & $61(51-71)$ & $66(56-76)$ \\
\hline $65-74$ years $(\%)$ & 13 & 21 & 27 \\
\hline$\geq 75$ years $(\%)$ & 11 & 19 & 28 \\
\hline Number of GP contacts in Intego (median (IQR)) & $2(1-4)$ & $6(4-9)$ & $11(8-15)$ \\
\hline Overall mortality rate /1000 & 11.3 & 7.1 & 12.7 \\
\hline Number of all-cause hospital admissions (mean (SD)) & $0.4(1.3)$ & $0.5(1.5)$ & $0.9(1.9)$ \\
\hline $\begin{array}{l}\text { Number of unplanned hospital admissions (mean } \\
\text { (SD)) }\end{array}$ & $0.06(0.29)$ & $0.07(0.33)$ & $0.19(0.53)$ \\
\hline 1. Uncomplicated hypertension & 14.8 & 31.2 & 41.1 \\
\hline 2. Lipid disorder & 11.6 & 22.4 & 28.8 \\
\hline 3. Psychological conditions & 12.1 & 20.5 & 28.7 \\
\hline 4. Malignancies & $7.4^{\dagger}$ & 16.7 & 27.3 \\
\hline 5. Osteoarthrosis & $7.4^{\dagger}$ & $13.9^{\dagger}$ & 19.0 \\
\hline 6. Asthma & $6.9^{\dagger}$ & $12.3^{\dagger}$ & 18.4 \\
\hline 7. Non-insulin- dependent diabetes & $9.7^{\dagger}$ & $15.3^{\dagger}$ & 18.0 \\
\hline 8. Dermatitis/atopic eczema & $6.1^{\dagger}$ & 10.8 & 16.5 \\
\hline 9. Back syndrome without radiating pain & $5.3^{\dagger}$ & $10.1^{\dagger}$ & 15.8 \\
\hline 4. Influenza vaccine & 6.0 & $26.9^{\dagger}$ & 48.1 \\
\hline 5. Platelet aggregation inhibitors & $3.1^{\dagger}$ & 20.5 & 37.7 \\
\hline 6. $\beta 1$-Selective beta blockers & $4.3^{\dagger}$ & $19.9^{\dagger}$ & 34.8 \\
\hline 7. Paracetamol & $5.0^{\dagger}$ & $17.3^{\dagger}$ & 34.7 \\
\hline 8. Corticosteroids (nasal) & $5.5^{\dagger}$ & $20.1^{\dagger}$ & 32.2 \\
\hline 9. Benzodiazepines & $3.3^{\dagger}$ & 13.1 & 27.8 \\
\hline 10. Opioid cough suppressants & $3.4^{\dagger}$ & 12.9 & 23.6 \\
\hline 11. Penicillin (broad spectrum) & $3.4^{\dagger}$ & 12.4 & 22.4 \\
\hline
\end{tabular}

Note: Values indicated with ${ }^{\dagger}$ are not on their correct position in top 10 according to their prevalence in that respective year.

Abbreviations: ED, Emergency Department; GP, general practitioner; HMG-CoA reductase, 3-hydroxy-3-methyl-glutaryl-CoA reductase; IQR, interquartile range; NSAIDs, non-steroidal anti-inflammatory drugs; PPIs, proton pump inhibitors; SD, standard deviation.

65 years and older was $40 \%$ in the $\mathrm{PP}_{5-9}$ group and $55 \%$ in the $\mathrm{PP}_{10+}$ group. The proportion of men was $51.3 \%$ in the nPP group, $46.2 \%$ in the $\mathrm{PP}_{5-9}$ group and $40.4 \%$ in the $\mathrm{PP}_{10+}$ group. The overall mortality rate was the highest in $\mathrm{PP}_{10+}$ patients (12.7/1000) and lowest in $\mathrm{PP}_{5-}$ ${ }_{9}$ patients (7.1/1000). The mean number of unplanned hospital admissions was 0.06 (SD 0.29) for nPP patients and 0.07 (SD 0.33) for
$\mathrm{PP}_{5-9}$ patients. For $\mathrm{PP}_{10+}$ patients, the mean number of unplanned hospital admissions was 0.19 (SD 0.53). Patients in the $\mathrm{PP}_{5-9}$ group had a median of 3 (IQR 1-5) registered chronic diseases compared to 4 (IQR 2-7) in the $\mathrm{PP}_{10+}$ group. Similar to the entire study cohort, NSAIDs dominated the lists in the nPP (18.2\%), $\mathrm{PP}_{5-9}(41.1 \%)$ and $\mathrm{PP}_{10+}(50.8 \%)$ groups. 
TABLE 3 Risk factors for polypharmacy

\begin{tabular}{|llll|}
\hline Effect & Point estimate (OR) & $95 \%$ Wald Cl & \\
\hline Age & 1.015 & 1.013 & 1.017 \\
\hline $\begin{array}{c}\text { Sex (female vs } \\
\text { male) }\end{array}$ & 1.161 & 1.108 & 1.216 \\
\hline $\begin{array}{c}\text { Number of chronic } \\
\text { diseases }\end{array}$ & 1.126 & 1.114 & 1.139 \\
\hline $\begin{array}{c}\text { Number of GP } \\
\text { contacts }\end{array}$ & 1.283 & 1.274 & 1.292 \\
\hline
\end{tabular}

Abbreviations: $\mathrm{Cl}$, confidence interval; GP, general practitioner; $\mathrm{OR}$, odds ratio.

\section{4 | Risk factors for polypharmacy}

A total of 39389 patients had a complete dataset for 2014 and 2015 and were included in the logistic regression analysis. In the univariate logistic regression analysis, all four variables had a value of $P<.001$ and were hence all included in the multivariable regression analysis (Table 3). A model fit was confirmed with significant results for the likelihood ratio test, Wald test and score test, that is $P$ values below .0001 . Age, female gender, number of chronic diseases and number of GP contacts all had a significant and positive association with polypharmacy. The odds of polypharmacy increased with $1.5 \%$ (95\% Cl: 1.013 to 1.017$)$ per 1-year increase in age. Women had $16.1 \%$ (95\% Cl: 1.108 to 1.216 ) increased odds of being prescribed at least five different medications compared to men. The presence of a new chronic disease increased the odds of polypharmacy with $12.6 \%$ (95\% Cl: 1.114 to 1.139). Every new GP contact increased the odds of polypharmacy with $28.3 \%$ ( $95 \% \mathrm{Cl}: 1.274$ to 1.292 ).

\section{4 | DISCUSSION}

Our Intego-IMA analysis provided a comprehensive report on the prevalence and evolution of polypharmacy and patient characteristics of both $\mathrm{PP}_{5-9}$ and $\mathrm{PP}_{10+}$ patients in Flanders. First, we found a significant increase in polypharmacy, despite years of rationalising medication use in primary healthcare. ${ }^{10}$ The prevalence rates of $\mathrm{PP}_{5-9}$ and $\mathrm{PP}_{10+}$ for patients aged 75 years and older increased significantly between 2011 and 2015. Furthermore, a majority of the patients aged $\geq 65$ years was prescribed at least five different medications. Second, $\mathrm{PP}_{5-9}$ and $\mathrm{PP}_{10+}$ patients appeared to differ to some extent from our observations regarding characteristics, medication use and outcomes. Third, the minimum intake of five medications was positively associated with age, female gender, number of chronic diseases and number of GP contacts.

Various qualities of our research contribute to the validity of the reported findings. The main strength was the access to a very large database consisting of real life data collected by GP's. Furthermore, our study population included adults from the age of 40 years with few exclusion criteria rendering our results representative for the general population. Finally, the cohort design allowed for a longitudinal evaluation of polypharmacy in our patient sample.
Conversely, we recognise that our study has some limitations. First, Intego only provided data on medications that were prescribed by primary healthcare physicians. At the time, there was however no database comprising information on dispensed medications. Consequently, no data from other physicians or relating to overthe-counter (OTC) medications were available for our analysis. We believe however that in our approach the prescription information is reliable. In practice, GP prescriptions will be influenced by other practitioners as they will often prolong medication prescriptions that have been initiated by other physicians (eg, during hospital stay); GPs have also access to all relevant information via specialist letters. Second, the actual medication intake and medication complexity was also not assessed due to the lack of robust measuring methods for adherence, inconclusive research on the association between polypharmacy and adherence and no availability of these data. ${ }^{19}$ Third, a medication count could be insufficient to identify high-risk patients as polypharmacy might not always be hazardous. ${ }^{12}$ Conversely, the retrospective nature of our study precluded us from assessing the appropriateness of medication use. Furthermore, the persistent relation between the number of medications and $\mathrm{MRH}$ has been extensively described and was recently reconfirmed as a relevant marker for future harm. ${ }^{1,20}$ While a rather simplistic measurement such as counting prescriptions is far from optimal, our study suggests it is still a useful metric to easily identify patients at high risk for negative clinical outcomes. We therefore aligned our definition of polypharmacy with the methodologies as proposed by van den Akker et al and Moriarty et al. ${ }^{21,22}$ In addition, we further specified this working definition by distinguishing between $\mathrm{PP}_{5}$ ${ }_{9}$ and $\mathrm{PP}_{10+}$ patients. Fourth, we did not have access to other risk factors for polypharmacy such as the level of income, level of education, obesity, living situation or self-medication hence these variables could not be analysed. ${ }^{4,5}$ Fifth, electronic prescribing was only made mandatory by law as of January $2020 .{ }^{23}$ The increased medication use could thus potentially be confounded by an incomplete implementation of electronic prescribing in our study. We believe, however, that Intego-associated prescribers are early-adopters of newly developed services meaning they had already largely implemented electronic prescribing at the start of our study period.

Our findings showed a prevalence of $29.5 \%$ for $\mathrm{PP}_{5-9}$ and $16.1 \%$ for $\mathrm{PP}_{10+}$ in 2015. Several investigations have reported a wide variation in prevalence due to using different definitions or evaluating other age categories. For example, 55.7\% of the Swedish patients and $46 \%$ of the American patients aged 65 years or older took at least five medications. ${ }^{24,25}$ But, only $22.8 \%$ of the patients aged 69 years or older living in the United Kingdom were considered polypharmacy patients. ${ }^{26}$ In Flanders, a total prevalence of 20\% in 2015 was found, regardless of age category. ${ }^{21}$ These results indicate that independently of using different definitions, polypharmacy remains common.

In our cohort, more than half of the patients had at least two chronic conditions. Uncomplicated hypertension was the most common chronic condition in all patients. However, based at ATC4 level, we found no antihypertensive drugs in the top 10 medication 
use, despite evidence-based guidelines and similar research. ${ }^{27,28} \mathrm{~A}$ potential association between underprescribing and polypharmacy has been previously reported and should be explored in subsequent studies. ${ }^{29-31}$ Furthermore, NSAIDs were prescribed at least once to $41.1 \%$ of the $\mathrm{PP}_{5-9}$ patients and to $50.8 \%$ of the $\mathrm{PP}_{10+}$ patients. The actual intake may even be an underestimation as ibuprofen in doses $<400 \mathrm{mg}$ is regarded as OTC medication in Belgium. Importantly, NSAIDs are contraindicated in patients with arterial hypertension, heart or renal failure and have been associated with unplanned hospital admissions. ${ }^{7,32}$ Serious drug events have also been ascribed to antiplatelet therapy and beta blockers, both of which were represented in the top $10 .^{7,33}$

We evaluated four risk factors that were positively associated with an increased risk for polypharmacy. In half of the $\mathrm{PP}_{10+}$ patients, GPs opened their EHR minimum once a month. This could have been the result of deteriorated health but also increases chances that newly developed symptoms will be noticed and treated accordingly. Consequently, more prescribed medications and hence a higher polypharmacy status could have been indicated. ${ }^{3}$ However, the association between more intense GP contact and a higher risk of polypharmacy is bidirectional. Polypharmacy is associated with increased $\mathrm{MRH}$ and this might in its turn lead to more frequent GP contacts. ${ }^{1}$ This was partly accounted for by counting GP contacts prior to polypharmacy status assessment. But patients already taking minimum five medications in 2014 were not excluded from the analysis. Furthermore, a recent study on factors related to the intake of minimum 15 medications found that a large majority of prescriptions was provided by the GP. ${ }^{34}$ While this might only concern a reflection of the current primary healthcare organisation, it also supports our findings regarding GP contacts being associated with polypharmacy, bearing in mind the potential bidirectional nature of this association. The association between chronic diseases and polypharmacy should be interpreted with caution as multimorbid patients treated with appropriate polypharmacy are not always at increased risk for medication-related harm. ${ }^{12}$

In this first paper of the larger PriMed research project, we aimed to characterise polypharmacy patients in primary healthcare. Multiple additional research hypotheses were identified following our analysis. Future research should focus on the two main observations that emerged from our study. First, we found no decrease and even a significant increase of patients taking minimum five medications between 2011 and 2015. Interventions such as computerised decision support provided to GP's or medication reviews and counselling performed in the community seem to have failed to impact overall medication use in primary healthcare. ${ }^{10}$ This could be partly attributed to a suboptimal identification of high-risk patients. Polypharmacy interventions such as deprescribing typically target older adults, but our results however suggest to not disregard younger patients by definition. ${ }^{10}$ The proportion of patients aged $\geq 75$ years with $\mathrm{PP}_{5-9}$ and $\mathrm{PP}_{10+}$ increased while simultaneously nearly half of the $\mathrm{PP}_{5-9}$ and $\mathrm{PP}_{10+}$ patients were still younger than 65 years. Patients between the ages of $40-65$ years often still have jobs and younger families, so the social impact of polypharmacy should not be underestimated. Furthermore, the idea that $\mathrm{PP}_{5-9}$ and $\mathrm{PP}_{10+}$ patients are different populations should be further scrutinised. Patient characteristics, drug therapy and outcomes in $\mathrm{PP}_{5}$. ${ }_{9}$ and $\mathrm{PP}_{10+}$ groups appeared to differ from our observations. For example, $\mathrm{PP}_{10+}$ patients had a lower percentage of men, the highest number of unplanned hospital admissions and the highest mortality rate. Age, female gender, number of chronic diseases and number of GP contacts were all positively associated with the intake of five or more medications. However, one of the few studies on risk factors for excessive polypharmacy found no association with female gender and even a significantly lower risk for patients aged 85 years and older. ${ }^{35}$ Both the ambiguous influence of age and the potential differences between $\mathrm{PP}_{5-9}$ and $\mathrm{PP}_{10+}$ patients should be further investigated and incorporated into research on target populations for polypharmacy interventions. In subsequent studies the determination of a meaningful cut off point of polypharmacy associated with clinical outcomes will be of value for the further optimisation of targeted interventions. Moreover, the effects on clinical outcomes of these interventions such as deprescribing should be further investigated. Second, a subsequent study should focus on medication use in both $\mathrm{PP}_{5-9}$ and $\mathrm{PP}_{10+}$ patients including possible under-treatment. The high number of potentially inappropriate medications in our top 10 is alarming and implies a significant risk of MRH such as unplanned hospital admissions in $\mathrm{PP}_{5-9}$ and $\mathrm{PP}_{10+}$ patients. $\mathrm{MRH}$ is persistently prevalent in older adults, as the association with polypharmacy is age dependent. ${ }^{1,8,9}$ Major risk factors for MRH such as old age and chronic diseases were present to varying degrees in our $\mathrm{PP}_{5-9}$ and $\mathrm{PP}_{10+}$ groups. Moreover, an underlying multifactorial aetiology of $\mathrm{MRH}$ demands a multidimensional treatment plan. ${ }^{9}$ This necessitates the integration of both a multidisciplinary approach in future interventions with communication between all relevant healthcare professionals and inclusion of patient preferences, shared decision making and adherence improvement strategies. In addition, interventions to reduce polypharmacy varied in outcome measurements. We propose to use the core outcome set specifically developed for multi-morbid, polymedicated older adults in future research on the improvement of medication use. ${ }^{36}$

\section{5 | CONCLUSION}

A large dynamic cohort study found an increase in medication use between 2011 and 2015. A growing proportion of $\mathrm{PP}_{5-9}$ and $\mathrm{PP}_{10+}$ patients aged 75 years and older was found with simultaneously around half of the $\mathrm{PP}_{5-9}$ and $\mathrm{PP}_{10+}$ patients being younger than 65 years. $\mathrm{PP}_{5-9}$ and $\mathrm{PP}_{10+}$ patients appeared to differ based on our observations of characteristics, drug therapy and outcomes. Older age, female gender, an increasing number of chronic diseases and more GP contacts were positively associated with polypharmacy.

\section{DISCLOSURES}

The authors declare that they have no conflict of interest. 


\section{AUTHOR CONTRIBUTIONS}

$\mathrm{LH}$ and $\mathrm{SN}$ contributed equally to this paper and share the first authorship. Concept/design: LH, SN, JH and LVDL; Statistics: SN; Data analysis/interpretation: $\mathrm{LH}, \mathrm{SN}, \mathrm{JH}$ and LVDL; Writing-original draft: $\mathrm{LH}, \mathrm{JH}$ and LVDL; Writing-critical revision: $\mathrm{LH}, \mathrm{SN}, \mathrm{JH}$, MVDA, GVP, XR, IS, BV, JT and LVDL. All authors have read and approved the final version of this manuscript.

\section{ORCID}

Laura Hellemans iD https://orcid.org/0000-0002-6787-9430

\section{REFERENCES}

1. Gnjidic D, Hilmer SN, Blyth FM, et al. Polypharmacy cutoff and outcomes: five or more medicines were used to identify community-dwelling older men at risk of different adverse outcomes. $J$ Clin Epidemiol. 2012;65(9):989-995.

2. Masnoon N, Shakib S, Kalisch-Ellett L, Caughey GE. What is polypharmacy? A systematic review of definitions. BMC Geriatr. 2017;17(1):230.

3. Boyd CM, Darer J, Boult C, Fried LP, Boult L, Wu AW. Clinical practice guidelines and quality of care for older patients with multiple comorbid diseases: implications for pay for performance. JAMA. 2005;294(6):716-724.

4. Carmona-Torres JM, Cobo-Cuenca AI, Recio-Andrade B, LaredoAguilera JA, Martins MM, Rodríguez-Borrego MA. Prevalence and factors associated with polypharmacy in the older people: 20062014. J Clin Nurs. 2018;27(15-16):2942-2952.

5. Slater N, White S, Venables R, Frisher M. Factors associated with polypharmacy in primary care: a cross-sectional analysis of data from The English Longitudinal Study of Ageing (ELSA). BMJ Open. 2018;8(3):e020270.

6. Hovstadius B, Petersson G. Factors leading to excessive polypharmacy. Clin Geriatr Med. 2012;28(2):159-172.

7. Leendertse AJ, Egberts AC, Stoker LJ, van den Bemt PM, HARM Study Group. Frequency of and risk factors for preventable medication-related hospital admissions in the Netherlands. Arch Intern Med. 2008;168(17):1890-1896.

8. Kouladjian L, Hilmer SN, Chen TF, Le Couteur DG, Gnjidic D. Assessing the harms of polypharmacy requires careful interpretation and consistent definitions. Br J Clin Pharmacol. 2014;78(3):670-671.

9. Stevenson JM, Davies JG, Martin FC. Medication-related harm: a geriatric syndrome. Age Ageing. 2019;49(1):7-11.

10. Rankin A, Cadogan CA, Patterson SM, et al. Interventions to improve the appropriate use of polypharmacy for older people. Cochrane Database Syst Rev. 2018;9:CD008165.

11. Van der Linden L, Hias J, Walgraeve K, Flamaing J, Tournoy J, Spriet I. Clinical pharmacy services in older inpatients: an evidence-based review. Drugs Aging. 2020;37(3):161-174.

12. Payne RA, Abel GA, Avery AJ, Mercer SW, Roland MO. Is polypharmacy always hazardous? A retrospective cohort analysis using linked electronic health records from primary and secondary care. Br J Clin Pharmacol. 2014;77(6):1073-1082.

13. Truyers C, Goderis G, Dewitte H, Van den Akker M, Buntinx F. The Intego database: background, methods and basic results of a Flemish general practice-based continuous morbidity registration project. BMC Med Inform Decis Mak. 2014;14:48.

14. IMA-AIM. Voorstelling; 2020. https://ima-aim.be/Voorstelling-17. Accessed May 4, 2020.

15. Knottnerus JA, Metsemakers J, Höppener P, Limonard C. Chronic illness in the community and the concept of 'social prevalence'. Fam Pract. 1992;9(1):15-21.
16. SAS Institute Inc. SAS/ETS® 13.2 User's Guide. Cary, NC: SAS Institute Inc.; 2014.

17. Walckiers D, Van der Heyden J, Tafforeau J. Factors associated with excessive polypharmacy in older people. Arch Public Health. 2015;73:50.

18. Sainani KL. Logistic regression. PM R. 2014;6(12):1157-1162.

19. Zelko E, Klemenc-Ketis Z, Tusek-Bunc K. Medication adherence in elderly with polypharmacy living at home: a systematic review of existing studies. Mater Sociomed. 2016;28(2):129-132.

20. De Vincentis A, Gallo P, Finamore P, et al. Potentially inappropriate medications, drug-drug interactions, and anticholinergic burden in elderly hospitalized patients: does an association exist with post-discharge health outcomes? Drugs Aging. 2020;37(8):585-593.

21. van den Akker M, Vaes B, Goderis G, Van Pottelbergh G, De Burghgraeve T, Henrard S. Trends in multimorbidity and polypharmacy in the Flemish-Belgian population between 2000 and 2015. PLoS One. 2019;14(2):e0212046.

22. Moriarty F, Hardy C, Bennett K, Smith SM, Fahey T. Trends and interaction of polypharmacy and potentially inappropriate prescribing in primary care over 15 years in Ireland: a repeated cross-sectional study. BMJ Open. 2015;5(9):e008656.

23. Koninklijk besluit betreffende het verplicht gebruik van het elektronisch voorschrift van geneesmiddelen voor ambulante patiënten, 2019012539 (May 5, 2019). http://www.ejustice.just. fgov.be/cgi_loi/change_lg.pl?language $=n|\&| a=N \& c n=2019050511$ \&table_name $=$ wet.

24. Morin L, Johnell K, Laroche ML, Fastbom J, Wastesson JW. The epidemiology of polypharmacy in older adults: register-based prospective cohort study. Clin Epidemiol. 2018;10:289-298.

25. Safran DG, Neuman P, Schoen C, Kitchman MS, Wilson IB, Cooper $B$, et al. Prescription drug coverage and seniors: findings from a 2003 national survey. Health Aff (Millwood). 2005;24(suppl. 1):W5-152-W5-166.

26. Rawle MJ, Richards M, Davis D, Kuh D. The prevalence and determinants of polypharmacy at age 69: a British birth cohort study. BMC Geriatr. 2018;18(1):118.

27. Williams B, Mancia G, Spiering W, et al. 2018 ESC/ESH Guidelines for the management of arterial hypertension: the Task Force for the management of arterial hypertension of the European Society of Cardiology and the European Society of Hypertension: The Task Force for the management of arterial hypertension of the European Society of Cardiology and the European Society of Hypertension. J Hypertens. 2018;36(10):1953-2041.

28. Wastesson JW, Cedazo Minguez A, Fastbom J, Maioli S, Johnell K. The composition of polypharmacy: a register-based study of Swedes aged 75 years and older. PLoS One. 2018;13(3):e0194892.

29. Fried TR, O'Leary J, Towle V, Goldstein MK, Trentalange M, Martin DK. Health outcomes associated with polypharmacy in community-dwelling older adults: a systematic review. J Am Geriatr Soc. 2014;62(12):2261-2272.

30. Kuijpers MA, van Marum RJ, Egberts AC, Jansen PA, OLDY (OLd people Drugs \& dYsregulations) Study Group. Relationship between polypharmacy and underprescribing. $\mathrm{Br} J$ Clin Pharmacol. 2008;65(1):130-133.

31. Wauters M, Elseviers M, Vaes B, et al. Too many, too few, or too unsafe? Impact of inappropriate prescribing on mortality, and hospitalization in a cohort of community-dwelling oldest old. Br J Clin Pharmacol. 2016;82(5):1382-1392.

32. Howard RL, Avery AJ, Slavenburg S, et al. Which drugs cause preventable admissions to hospital? A systematic review. Br J Clin Pharmacol. 2007;63(2):136-147.

33. Budnitz DS, Lovegrove MC, Shehab N, Richards CL. Emergency hospitalizations for adverse drug events in older Americans. N Engl J Med. 2011;365(21):2002-2012. 
34. Castro-Rodríguez A, Machado-Duque ME, Gaviria-Mendoza A, Medina-Morales DA, Álvarez-Vera T, Machado-Alba JE. Factors related to excessive polypharmacy ( $\geq 15$ medications) in an outpatient population from Colombia. Int J Clin Pract. 2019;73(3): e13278.

35. Rieckert A, Trampisch US, Klaaßen-Mielke R, et al. Polypharmacy in older patients with chronic diseases: a cross-sectional analysis of factors associated with excessive polypharmacy. BMC Fam Pract. 2018;19(1):113.

36. Beuscart J-B, Knol W, Cullinan S, et al. International core outcome set for clinical trials of medication review in multi-morbid older patients with polypharmacy. BMC Med. 2018;16(1):21.

\section{SUPPORTING INFORMATION}

Additional Supporting Information may be found online in the Supporting Information section.

How to cite this article: Hellemans L, Nuyts S, Hias J, et al. Polypharmacy and excessive polypharmacy in communitydwelling middle aged and aged adults between 2011 and 2015. Int J Clin Pract. 2020;00:e13942. https://doi. org/10.1111/ijcp.13942 